\title{
Improving the Effectiveness of Medication Review: Guidance from the Health Literacy Universal Precautions Toolkit
}

\author{
Barry D. Weiss, MD, Angela G. Brega, PhD, William G. LeBlanc, PhD, \\ Natabbona M. Mabachi, MPH, PhD, Juliana Barnard, MA, Karen Albright, PhD, \\ Maribel Cifuentes, RN, Cindy Brach, MPP, and David R. West, PhD
}

Background: Although routine medication reviews in primary care practice are recommended to identify drug therapy problems, it is often difficult to get patients to bring all their medications to office visits. The objective of this study was to determine whether the medication review tool in the Agency for Healthcare Research and Quality Health Literacy Universal Precautions Toolkit can help to improve medication reviews in primary care practices.

Methods: The toolkit's "Brown Bag Medication Review" was implemented in a rural private practice in Missouri and an urban teaching practice in California. Practices recorded outcomes of medication reviews with 45 patients before toolkit implementation and then changed their medication review processes based on guidance in the toolkit. Six months later we conducted interviews with practice staff to identify changes made as a result of implementing the tool, and practices recorded outcomes of medication reviews with 41 additional patients. Data analyses compared differences in whether all medications were brought to visits, the number of medications reviewed, drug therapy problems identified, and changes in medication regimens before and after implementation.

Results: Interviews revealed that practices made the changes recommended in the toolkit to encourage patients to bring medications to office visits. Evaluation before and after implementation revealed a 3 -fold increase in the percentage of patients who brought all their prescription medications and a 6-fold increase in the number of prescription medications brought to office visits. The percentage of reviews in which drug therapy problems were identified doubled, as did the percentage of medication regimens revised.

Conclusions: Use of the Health Literacy Universal Precautions Toolkit can help to identify drug therapy problems. (J Am Board Fam Med 2016;29:18-23.)

Keywords: Education of Patients, Health Literacy, Medical Errors, Polypharmacy

Drug therapy problems are common in primary care; they often are related to prescribing errors by clinicians as well as polypharmacy. ${ }^{1-3}$ Guide-

This article was externally peer reviewed.

Submitted 18 May 2015; revised 28 August 2015; accepted 9 September 2015.

From the Department of Family and Community Medicine, University of Arizona, Tucson (BDW); the Department of Community and Behavioral Health, Colorado School of Public Health, Aurora (AGB, KA); the Department of Family Medicine, University of Colorado Anschutz Medical Campus, Aurora (AGB, WGL, MC, DRW); the American Academy of Family Physicians, Kansas City, KS (NMM); the Colorado Health Outcomes Program, University of Colorado Anschutz Medical Campus, Aurora (JB, KA, DRW); and the Center for Delivery, Organization, and lines have been developed to help reduce prescribing errors and polypharmacy by encouraging routine reviews of patients' medication regimens. $^{4-7}$ However, effective use of these guidelines requires that patients provide accurate

Markets, Agency for Healthcare Research and Quality, Rockville, MD (CB).

Funding: Support for this work was provided by a contract from the Agency for Healthcare Research and Quality (HHSA290200710008 to DRW).

Conflict of interest: none declared.

Corresponding author: Barry D Weiss, MD, Department of Family and Community Medicine, University of Arizona, 1450 N. Cherry Ave, Tucson, AZ 85724 (E-mail: bdweiss@ u.arizona.edu). 
Table 1. Tools/Topics Included in the Health Literacy Universal Precautions Toolkit

How to form a team to assess the practice and raise awareness about health literacy

Tools for improving spoken communication

How to communicate clearly

How to use teach-back

Effective follow-up

Effective phone communications

"Brown bag" medication review

How to address language differences

Dealing with patients from different cultures

Tools for improving written communication

How to design easy-to-read material

How to use health education material effectively

Good signage

Improving patient self-management and empowerment

Encouraging patients to ask questions

Helping patients to make action plans

Improving medication adherence and accuracy

Getting patient feedback

Improving support systems

Linking patients to nonmedical support services

Linking patients to medication resources

Linking patients to health and literacy resources in the community

Adapted from ref. 11.

information to clinicians about the medications they are taking.

The "brown bag medicine review" is a practice in which patients aid in medication reviews by putting all their medications in a bag and bringing them to their clinician for review. ${ }^{8,9}$ It can, however, be difficult to get patients to bring all their medications to office visits, limiting the value of the resulting reviews. ${ }^{10}$

The Health Literacy Universal Precautions Toolkit, developed by the Agency for Health care Research and Quality (AHRQ), was designed to help clinicians improve communication with and support for patients of all health literacy levels. ${ }^{11}$ The tools in the toolkit are listed in Table 1. One of the tools in the toolkit ("Brown Bag Medication Review") provides guidance on effectively implementing medication reviews, including approaches to encourage patients to bring medications to office visits.

In this article we report findings related to implementation of the Brown Bag Medication Review tool in 2 family medicine practices as part of a national demonstration of the toolkit. Our primary objective was to assess whether use of this tool helped those practices improve the rate with which patients bring all their medications to office visits for review. A second objective was to investigate the percentage of patients who had drug therapy problems identified during the reviews and whose medication regimens were modified as a result of reviews performed before and after implementation of the tool.

\section{Methods}

In 2013 to 2014, AHRQ supported a national demonstration of the toolkit's use. As part of the demonstration, a subset of tools from the toolkit was implemented within a diverse sample of 12 primary care practices. Each practice conducted a self-assessment and selected from the toolkit tools to implement over a 6-month period.

Two of the practices, both from the American Academy of Family Physicians' National Research Network, implemented the Brown Bag Medication Review tool. One practice is a medium-sized family medicine practice certified as a patient-centered medical home in rural Missouri. This practice provides care mainly for white patients (95\%); approximately one third of patients are older adults, and one third receives care through Medicaid. The other practice is a large federally qualified health center and family medicine residency teaching program in southern California. This practice cares mainly for Hispanic patients and patients receiving care through Medicaid (75\%). More information about the practices is provided in Table 2.

Both practices implemented all the recommended action steps from the Brown Bag Medication Review tool (Table 3). Guidance in those action steps focuses on getting patients to bring their medications to office visits, conducting the medication review, confirming patient understanding of medication regimens, clarifying medications and medication instructions, identifying drug therapy problems (eg, unnecessary drug therapy, incorrect dosages, duplicate medications), ${ }^{12}$ documenting review results, and providing an updated medication list to patients. While the action steps are designed to enhance a clinician's ability to accurately determine what medications their patients are taking and to identify drug therapy problems, the medication review 
Table 2. Characteristics of the Two Practices That Implemented the Brown Bag Medication Review Tool

\begin{tabular}{|c|c|c|}
\hline Characteristic* $^{*}$ & Missouri Practice & California Practice \\
\hline Practice type & $\begin{array}{l}\text { Private family medicine practice; } \\
\text { patient-centered medical home }\end{array}$ & $\begin{array}{l}\text { Family medicine residency; federally qualified } \\
\text { community health center }\end{array}$ \\
\hline Location & Rural & Suburban \\
\hline \multicolumn{3}{|l|}{ Clinicians $(\mathrm{n})^{\dagger}$} \\
\hline Full time & 7 & 4 \\
\hline Part time & 0 & 30 \\
\hline Patient population (approximate $n$ ) & 4,700 & 10,000 \\
\hline \multicolumn{3}{|l|}{ Patient sociodemographics (\%) } \\
\hline Medicaid & 35 & 75 \\
\hline Black & 1 & 5 \\
\hline White & 95 & 10 \\
\hline Hispanic & 2 & 79 \\
\hline Age $>65$ years & 30 & 12 \\
\hline Non-English-speaking & 1 & 50 \\
\hline Limited health literacy ${ }^{\ddagger}$ & 32 & 56 \\
\hline
\end{tabular}

*Data on practice characteristics were provided by the individual practices.

${ }^{\dagger}$ Clinicians include physicians, advanced practice nurses, physician assistants.

${ }^{\ddagger}$ Percentage of patents with limited health literacy was based on the Health Literacy Prevalence Calculator, which uses practice demographics to estimate the prevalence of limited health literacy among a patient population (http://surroundhealth.net/ Topics/Education-and-Learning-approaches/Health-literacy/Articles/Health-literacy-A-foundation-to-effective-patient/HealthLiteracy-Prevalence-Calculator.aspx).

tool is not specifically designed for medication reconciliation when patients undergo transitions of care.

The study protocol was approved by the institutional review boards of the University of Colorado, the American Academy of Family Physicians, and the institution overseeing research at the residency training program in California. Separate institutional review board review was not required for the Missouri practice.

\section{Interviews}

We interviewed practice staff during site visits made before and after implementation of the tool and through 4 phone calls made during implementation. Both the telephone and site-visit interviews used open-ended questions to query practice staff about changes the practice made in the process of implementing the tool. Specifically, we asked about how they implemented each of the tools, who implemented them, and problems/obstacles they encountered during implementation.

\section{Outcome Data Collection}

Each practice was asked to record data for at least 20 medication reviews conducted before imple- menting the practice changes outlined in Table 3 , and for at least 20 medication reviews conducted after the conclusion of the 6-month implementation period. In conducting reviews, practices were instructed to select patients from among those identified during routine clinical practice (eg, the prescription refill process, regular follow-up visits) as requiring a full review of current medications.

During or shortly after each patient's visit, clinicians conducting the medicine review completed a data collection form recording (1) the number of medicines brought to the visit, (2) whether the patient reported that all medicines had been brought to the visit, (3) the number of medicines reviewed during the visit, (4) whether problems were found with the patient's medicine regimen (eg, duplicate medications, expired medications), and (5) whether changes to the medication regimen were made as a result of the review. The data collection form was designed by clinicians with experience conducting medication reviews, and it was subsequently reviewed and approved by a panel of physician consultants to ensure that all pertinent information was being collected. There was, however, no formal pilot testing or validation of this data collection form. 
Table 3. Toolkit's Action Plan for Brown Bag Medication Review

Ways to remind patients to bring medications
Appointment cards
During appointment reminder calls
During office visits
Posters in exam rooms and waiting rooms
Bulletin board display of anonymous cases that make the
case for bringing medications
Emphasize that the review usually results in taking fewer
medications
Provide a carrier (bag or sack) in which patients can bring
medications to visits
What to tell patients to bring
All prescription medications including pills and creams
All over-the-counter medicines taken regularly
All vitamins and supplements
All herbal medications
What to do during the medication review
Offer praise for bringing the medications
Track progress
Track the percentage of patients each day who have had a
medication review completed
Aim for $90 \%$ of patients to have a review over a 12-month
period

Adapted from ref. 11.

\section{Data Analysis}

Interviews were recorded and reviewed by project staff to ensure that we had an accurate record of the practice staff's responses to interview questions. We did this for both the telephone and in-person interviews.

For outcome data, we compared data collected during medication reviews performed before and those performed after tool implementation using SAS version 9.4 (SAS, Inc., Cary, NC). Independent sample $t$ tests were used to compare continuous variables (ie, how many medications were brought to visits, the number of medications reviewed during visits). $\chi^{2}$ Analyses were used to compare dichotomous variables (ie, whether all medications were brought to visits, whether problems were found, whether changes were made in the medication regimen).

\section{Results}

\section{Interviews}

Practice staff identified the changes made to their clinical and administrative procedures as part of their implementation efforts. Notable among these were (1) modifying electronic medical records sys- tems to allow staff to note that medication reviews had occurred, (2) ordering reusable bags to distribute to patients for carrying medications to appointments, and (3) enlisting all practice staff in reminding patients to bring medications to office visits. The residency practice in California also encouraged supervising physicians to incorporate the conduct of medication reviews into their evaluation of residents' performance. The major challenge they encountered was identifying methods of billing for medication reviews.

At the rural Missouri practice, the medication reviews themselves were performed by nurses when they brought patients to the examination room. At the California teaching practice, resident physicians performed the medication reviews during their encounters with patients.

\section{Outcomes}

Practices provided medication review data for 45 patients before implementation and 41 patients following implementation of the tool. Key results for both the individual practices and the 2 practices combined are summarized in Table 4.

From the time point before to that after implementation, there was more than a 3 -fold increase (from $20.0 \%$ to $68.3 \%$ ) in the percentage of patients undergoing a medication review who reported that they had brought all their prescription medicines to the office visit $\left(\chi^{2}=27.4 ; \mathrm{df}=2 ; P<\right.$ $.001)$. Perhaps as a result of this increase, there was a 6-fold increase in the number of prescription medications brought to visits. Before implementation of the toolkit, patients brought an average of 1 medication to office visits; after implementation the number increased to $6.8(t=7.28 ; \mathrm{df}=57.9 ; P<$ $.001)$. There was also an $84 \%$ increase in the number of prescription medications reviewed, from an average of 3.3 before implementation to 6.1 after implementation $(t=3.03 ; \mathrm{df}=75 ; P<.003)$. Likewise, the percentage of patients who reported bringing all their nonprescription medications to office visits (ie, over-the-counter medicines and supplements) more than doubled, from $9.1 \%$ to $19.5 \%\left(\chi^{2}=15.8 ; \mathrm{df}=4 ; P=.003\right)$.

The percentage of patients for whom the medication review identified problems with the medication regimen almost doubled from $17.8 \%$ before implementation to $34.2 \%$ after implementation, although this change was not statistically significant $\left(\chi^{2}=3.0 ; \mathrm{df}=1 ; P=.082\right)$. There was, however, 
Table 4. Comparison of Medication Reviews Performed Before and After Implementation of the Brown Bag Medication Review Tool

\begin{tabular}{|c|c|c|c|c|c|c|c|}
\hline \multirow[b]{2}{*}{ Item Evaluated } & \multicolumn{3}{|c|}{ Before Implementation } & \multicolumn{3}{|c|}{ After Implementation } & \multirow[b]{2}{*}{$\begin{array}{c}P \\
\text { Value }\end{array}$} \\
\hline & $\begin{array}{l}\text { Missouri Clinic } \\
\quad(\mathrm{n}=27)\end{array}$ & $\begin{array}{l}\text { California Clinic } \\
\quad(\mathrm{n}=18)\end{array}$ & $\begin{array}{c}\text { Total } \\
(\mathrm{n}=45)\end{array}$ & $\begin{array}{l}\text { Missouri Clinic } \\
\quad(\mathrm{n}=20)\end{array}$ & $\begin{array}{l}\text { California Clinic } \\
\quad(\mathrm{n}=21)\end{array}$ & $\begin{array}{c}\text { Total } \\
(\mathrm{n}=41)^{*}\end{array}$ & \\
\hline $\begin{array}{l}\text { Prescription medications } \\
\text { brought to office visit, } \\
\text { mean (SD) }\end{array}$ & $0.44(1.9)$ & $1.8(2.6)$ & $1.0(2.3)$ & $8.0(5.4)$ & $5.6(3.4)$ & $6.8(4.6)$ & $<.001$ \\
\hline $\begin{array}{l}\text { Prescription medications } \\
\text { reviewed with patient, } \\
\text { mean (SD) }\end{array}$ & $3.25(5.4)$ & $3.3(2.4)$ & $3.3(4.3)$ & $7.4(4.5)$ & $4.9(3.1)$ & $6.1(4.0)$ & .003 \\
\hline $\begin{array}{l}\text { All prescription } \\
\text { medications brought } \\
\text { to visit (patient } \\
\text { report) }\end{array}$ & 7.4 & 38.9 & 20 & 70.0 & 66.7 & 68.3 & $<.001$ \\
\hline $\begin{array}{l}\text { All nonprescription } \\
\text { medications brought } \\
\text { to visit (patient } \\
\text { report) }\end{array}$ & 11.1 & 5.9 & 9.1 & 25.0 & 14.3 & 19.5 & .003 \\
\hline $\begin{array}{l}\text { Problems found with } \\
\text { medication regimen }\end{array}$ & 25.9 & 5.6 & 17.8 & 40.0 & 28.6 & 34.2 & .082 \\
\hline $\begin{array}{l}\text { Changes made to } \\
\text { medication regimen as } \\
\text { a result of the review }\end{array}$ & 25.9 & 5.6 & 17.8 & 50.0 & 33.3 & 41.5 & .016 \\
\hline
\end{tabular}

SD, standard deviation. Data are percentages unless otherwise indicated.

a significant increase in the percentage of patients whose medication regimens were changed as a result of the review $\left(17.8 \%\right.$ vs $41.5 \% ; \chi^{2}=5.8 ; \mathrm{df}=$ $1 ; P=.016)$.

\section{Discussion}

Our results suggest that use of the Brown Bag Medication Review tool in the AHRQ Health Literacy Toolkit can help family medicine practices improve the effectiveness of their medication review processes. Implementing the tool resulted in a 3 -fold increase in the percentage of patients who reported bringing all their medications to office visits, a 6-fold increase in the actual number of medications brought to visits, and a $84 \%$ increase the percentage of patients bringing all their medications to office visits. It also resulted in more than double the rate at which patients had changes made to their medication regimens as a result of the medication reviews. Improvements in the medication review process occurred in both clinics, even though they used different approaches: medication reviews were conducted by nurses in the Missouri clinic and by physicians in the California clinic.

Given the high rate of drug therapy problems in clinical practice, ${ }^{1,2}$ any intervention that can achieve such improvements is noteworthy. Indeed, we are unaware of other interventions that have achieved this degree of success.

Nonetheless, our results should be considered preliminary for several reasons. First, data before and after implementation were based on self-report by clinicians and patients, so their accuracy cannot be verified. Second, when clinicians selected patients for medication reviews, they may have selected patients who were easier to work with or whom they considered to be more likely to bring medications to office visits. The extent to which this occurred cannot be determined. Third, it is possible that prescribing habits changed over the course of the 6-month implementation period; the increased number of medications brought to office visits could have reflected an increase in the total number of medications being prescribed to patients. While possible, we believe this is unlikely.

Another concern is that while there was a clear improvement in the percentage of patients reporting that they brought their medications to office visits, those percentages are still not optimal. For example, even after tripling the percentage of patients who reported bringing all their prescription medications to visits, only $68.3 \%$ did so. Even after doubling the percentage of patients who reported bringing all their nonprescription medications to 
visits, only $19.5 \%$ did so. Thus, while implementing the toolkit improved the rate at which patients brought in their medications, based on our results the toolkit cannot be viewed as a complete solution to improving medication reviews.

Most important, our findings are based on a small sample of patients from 2 practices who were motivated to improve their medication review processes. Thus the results cannot be generalized to all practices. However, significant improvements were noted, even with small sample sizes that provided limited statistical power, and we believe the results can be generalized to practices that are similarly motivated to improve their medication review efforts. For such motivated practices, the toolkit may provide a readily available, low-cost approach to identifying drug therapy problems.

\section{References}

1. Prescribing errors are the most common medication errors in primary care practices: Research activities, February 2009, no. 374. Rockville, MD: Agency for Healthcare Research and Quality. Available from: http://archive.ahrq.gov/news/newsletters/researchactivities/feb09/0209RA5.html. Accessed November $11,2015$.

2. Kuo GM, Phillips RL, Graham D, Hickner JM. Medication errors reported by US family physicians and their office staff. Qual Saf Health Care 2008;17: 289-90.

3. Johnell K, Klarin I. The relationship between number of drugs and potential drug-drug interactions in the elderly: a study of over 600,000 elderly patients from the Swedish Prescribed Drug Register. Drug Saf 2007;30:911-8.
4. American Geriatrics Society 2012 Beers Criteria Update Expert Panel. American Geriatrics Society updated Beers Criteria for potentially inappropriate medication use in older adults. J Am Geriatr Soc 2012;60:616-31.

5. Gallagher P, O'Mahony D. STOPP (Screening Tool of Older Persons' potentially inappropriate Prescriptions): application to acutely ill elderly patients and comparison with Beers' criteria. Age Ageing 2008; 37:673-9.

6. Wolff CM, Nowacki AS, Yeh JY, Hickner JM. A randomized controlled trial of two interventions to improve medication reconciliation. J Am Board Fam Med 2014;27:347-55.

7. Varkey P, Cunningham J, Bisping DS. Improving medication reconciliation in the outpatient setting. Jt Comm J Qual Patient Saf 2007;33:286-92.

8. Nathan A, Goodyer L, Lovejoy A, Rashid A. "Brown bag" medication reviews as a means of optimizing patients' use of medication and of identifying potential clinical problems. Fam Pract 1999;16:278-82.

9. Sarzynski EM, Luz CC, Zhou S, Rios-Bedoya CF. Medication reconciliation in an outpatient geriatrics clinic: does accuracy improve if patients "brown bag" their medications for appointments? J Am Geriatr Soc 2014;62:567-9.

10. Brown S. Overcoming the pitfalls of medication reconciliation. Nurs Manage 2012;43:15-7.

11. DeWalt DA, Callahan LF, Hawk VH, et al. Health Literacy Universal Precautions Toolkit. AHRQ publication no. 10-0046-EF. Rockville, MD: Agency for Healthcare Research and Quality; April 2010.

12. Cipolle RJ, Stands LM, Morley PC. Drug therapy problems. In: Pharmaceutical care practice: the patient centers approach to medication management services, 3rd ed. New York: McGraw-Hill Companies; 2012:141-82. 